\title{
Análisis del Sistema de Estadística de la Balanza Comercial de México
}

\author{
Analysis of the Statistics System of the Mexican Trade Balance
}

\author{
Germán Martínez Prats \\ germanmtzprats@hotmail.com \\ Universidad Juárez Autónoma de Tabasco \\ México \\ https://orcid.org/0000-0001-6371-448X
}

Recibido: 12 de noviembre del 2018

Aprobado: 05 de diciembre del 2018

\section{RESUMEN}

La información del intercambio comercial de mercancías que se realiza en México se compila a través del Instituto Nacional de Estadística y Geografía (INEGI) el cual registra la Estadística de Comercio Exterior de México (ECEM), cuya información permite la toma de decisiones en materia comercial y financiera. Sin embargo, no se ha desarrollado un método que permita validar los datos de volumen importado y exportado de las operaciones comerciales mediante un agente aduanal, por lo que existe un sobre registro de los datos. El objetivo de la presente investigación es establecer un método de validación de las importaciones y exportaciones considerando los volúmenes por fracción arancelaria y unidad de medida, así como los Incoterms, para generar un registro específico que permita obtener información más confiable y sirva para la toma decisiones de los actores involucrados en el comercio exterior. Dicho análisis se desarrolló con un enfoque cuantitativo con información de pedimentos del 2012 al 2015 obtenida por agencias aduanales en México.

Descriptores: Importación; exportación; análisis económico; comercio internacional; balanza comercial.

\section{ABSTRACT}

The information of the commercial exchange of merchandise carried out in Mexico is compiled through the National Institute of Statistics and Geography (INEGI) which registers the Statistics of Foreign Trade of Mexico (ECEM), whose information allows the decision-making in commercial and financial matters. However, a method that allows the validation of volume data imported and exported from commercial operations through a customs broker has not been developed, so there is an overegistration of the data. The objective of this paper is to establish a method of validation of imports and exports considering the volumes by tariff fraction and unit of measure, as well as the Incoterms, to generate a specific registry that allows obtaining more reliable information and serves to make decisions of the actors involved in foreign trade. This analysis was 
developed with a quantitative approach with information from 2012 to 2015 obtained by customs agencies in Mexico.

Descriptors: Importation; exportation; economic analysis; international trade; trade balance.

\section{INTRODUCCIÓN}

El registro de las operaciones de las mercancías en relación a la importación y exportación datan de la época colonial. A partir de 1888, se elabora un registro formal mediante la Dirección General de Estadística (DGE). En 1980, se actualizó la Clasificación Industrial Internacional Uniforme (CIIU) para la presentación de resultados según la actividad económica de origen y principales productos. Asimismo, se instrumentó el Sistema Armonizado de Designación y Codificación de Mercancías (SA) con el cual se implementó una nomenclatura arancelaria lo que propició la presentación de cifras homogéneas entre los países. Para 2005 el CIIU fue reemplazado por la publicación de los Principales Productos del SA (Instituto Nacional de Estadística y Geografía [INEGI], 2018).

En nuestro país se cuentan con registros de la Balanza Comercial a partir de 1991, aunque existe información a partir de 1938, contándose cada vez con datos más específicos de los diferentes rubros que integran dicha balanza. Con el paso de los años, las innovaciones tecnológicas y conceptuales se han convertido en la principal herramienta de mejora, lo que ha contribuido notablemente en la integración y procesamiento de los datos estadísticos.

Las fuentes primarias de información de la balanza comercial de mercancías de México, son los registros de entrada y/o salida de las operaciones comerciales que se llevan a cabo en cada una de las aduanas. Para su control, el Servicio de Administración Tributaria (SAT) ha implementado el Sistema de Automatización Aduanera Integral (SAAI M3), que consiste en un proceso de validación de pedimentos aduaneros que da lugar a un intercambio de información entre agentes y apoderados aduanales, por una parte, y la autoridad aduanera por la otra. 
La estadística está basada en la Tarifa de la Ley de Impuestos Generales de Importación y Exportación (TIGIE) e incluye las exportaciones e importaciones de mercancías para 22 secciones, 98 capítulos, 1250 partidas, 5325 subpartidas y 12685 fraciones arancelarias aproximadamente. Es importante, destacar que, tanto en la exportación como en la importación, el INEGI incorpora la Sección XXIII con la finalidad de incluir el capítulo 99 y la fracción 99.99.99.99 (Códigos arancelarios no catalogados en la tarifa), todas aquellas operaciones de comercio exterior que desde su origen presentan inconsistencias de captura o registro, y por lo tanto no se pueden clasificar en códigos específicos de la TIGIE.

Asimismo, se presentan códigos arancelarios a 6 dígitos que se utilizan para describir operaciones especiales y se podrán identificar con la descripción siguiente: «Operaciones especiales reportadas en códigos a 6 dígitos», facilitando a los usuarios la agregación o acumulación de cifras correspondientes al valor de las mercancías a nivel de 2, 4, 6 y 8 dígitos.

Las exportaciones se valoran FOB (Free on Board), que en español es equivalente a Franco a Bordo y se refiere al valor de venta de las mercancías en la aduana de salida. Asimismo, las importaciones se valoran FOB, de acuerdo con el valor comercial de compra de las mercancías en el lugar de procedencia, por lo que el importe de fletes, seguros y demás gastos incurridos para hacer llegar la mercancía a la aduana de entrada, se consignan en un renglón por separado. Lo anterior, explica que la suma de las importaciones FOB, más los gastos de fletes y seguros dan como resultado las importaciones CIF (Cost, Insurance and Freight), que en español es equivalente a Costos, Seguros y Fletes.

\section{DESARROLLO}

\section{Antecedentes de los registros en materia de Balanza Comercial en México}

En casi todos los países del mundo, el crecimiento económico reflejado en el PIB fue mayor durante los años comprendidos entre 1950-1973 que en años anteriores o posteriores. En México esta etapa fue mayor, ya que abarcó más de 40 años, desde 
1939 a 1981, como referencia el PIB creció cuatro veces más que la población, ese gran crecimiento económico, redujo de manera significativa la diferencia en materia de ingreso entre los Estados Unidos de América y nuestro país, expresado en dólares constantes, en 1939 el PIB per cápita en México alcanzaba 22\% del promedio en la nación vecina y 30\% en 1981 (Moreno-Brid, 1998).

México se ubicaba entre las economías más grandes de América Latina al final de este periodo, gracias a que el ritmo de crecimiento de la economía mexicana fue aún mayor durante los últimos cinco años de ese periodo, para finales de la década de los setenta, su desarrollo fue de la mano de una ambiciosa política comercial de industrialización, las exportaciones petroleras sirvieron como fuente de financiamiento y la adquisición de deuda pública como segundo elemento de financiamiento, es en este punto donde se genera la apertura comercial de nuestro país, y de este se deriva la importancia del análisis de la balanza comercial.

Derivado del aumento en importaciones al finalizar la Segunda Guerra Mundial, que dió como resultado una crisis de la balanza de pagos a finales de la década de los cuarenta, se aplicó el sistema de protección a la industria nacional, dirigido principalmente a la producción de bienes de consumo (Carrillo, 2004).

México con la caída del precio del petróleo, ejecutó la estrategia de la liberación comercial para crecer con las exportaciones, especialmente en las manufactureras. Como consecuencia se firmó el Acuerdo General sobre Aranceles Aduaneros y Comercio (GATT), y finalizó con la firma del Tratado de Libre Comercio del América de Norte (TLCAN). Gracias a este tratado, en 1980 las exportaciones de productos manufactureros eran del 20\% y en 2003 tuvo un incremento al 85\% (Cardero, Mántey, \& Mendoza, 2006).

Las exportaciones y la inversión extranjera directa se han concentrado en un pequeño grupo de empresas, sectores y estado del país, y tras este proceso, el más afectado ha sido el sector agrícola, ya que las inversiones para este rubro se han visto disminuidas. La tendencia a la globalización económica, la información de tipo estadística permite conocer el desarrollo comercial del país, mediante el estudio del desempeño de sus 
sectores económicos, y muestra por otra parte el grado de consolidación de sus estructuras comerciales y económicas.

Estos datos son utilizados como insumos para la elaboración de:

a) El sistema de cuentas nacionales de México.

b) Reportes de desempeño y elaboración de perfiles de las empresas manufactureras de exportación.

c) Importaciones y exportaciones por estado.

d) Anuario estadístico.

Adicionalmente será un insumo fundamental para el análisis y medición en las cadenas globales de valor.

Para la elaboración de la balanza comercial, las operaciones a ser consideradas para la obtención de información estadística, son las operaciones comerciales que realizan las personas físicas y morales, y que quedan registradas a través de un pedimento ya sea de importación o exportación.

En el proceso de elaboración de la balanza comercial son aplicados diversos sistemas informáticos diseñados por la Subdirección de Servicios de Desarrollo de Sistemas, y así se generan diversos productos estadísticos que permiten la atención de requerimientos de los distintos usuarios de la información, el sistema en conjunto se llama Sistema Integral Comercio Exterior (SICE), e integra las bases de datos que se general mes con mes y parte primordial en la presentación de la balanza comercial.

EI SICE permite el uso y aprovechamiento de la información, principalmente de información a nivel registro, y en términos generales, considera los siguientes puntos:

a) Datos en valor y volumen de las operaciones de comercio exterior.

b) Origen, destino y datos referentes al origen de las mercancías.

c) Información obtenida del sistema armonizado de designación y codificación de mercancías, fracción, partida, capitulo, etc.

d) Numero de Aduana en la que se llevó a cabo la operación y seccione aduanera a la que pertenece.

e) Medio de transporte. 
f) INCOTERM utilizado

\section{Difusión de información y atención de requerimientos}

El mantenimiento de la calidad de la Información utilizada se considera un elemento vital para otorgar confianza en información generada por el INEGI, por lo cual se ha desarrollado un sistema integral del aseguramiento de la calidad de los procesos de generación, integración y difusión de la información estadística y geográfica, en el cual se aplican las mejores prácticas internacionales en la materia, dichas practicadas son generadas y aplicadas por organismos internacionales, los cuales han desarrollado procesos, normas, lineamientos, sistemas, procedimientos e instrumentos que han probado su eficacia para el aseguramiento de la calidad en la generación, integración y difusión de la información estadística y geográfica.

Estos han sido avances importantes, pero aun así no se alcanza la estandarización de los volúmenes de las mercancías que conforman la balanza comercial, pues el sistema no considera como un insumo primordial la fracción arancelaria, y al tratarse de un mismo producto, se puede llegar a clasificar en distintas fracciones, pero una misma fracción representa siempre el mismo producto, tampoco toma en cuenta el régimen aduanero, si es temporal, definitivo, en tránsito, generándose así discrepancias en la información obtenida, son estos elementos que permitirán la estandarización de los volúmenes.

\section{Método de validación de los datos de la Balanza Comercial}

El método de validación consiste en agrupar todas las operaciones por tipo ya sean de importación o exportación, de esta forma se determina si es ingreso o egreso, como segunda acción es agruparlas por fracción arancelaria, esto permite conocer a detalle el monto importado o exportado de una fracción en específico, es también requerido se considere el Incoterm utilizado en el pedimento de exportación, ya que es aquí donde se genera la validación de la información. 
En la forma de cálculo actual todas las exportaciones se consideran FOB (Franco a Bordo), de acuerdo al Mexico International Chamber of Commerce (2010), este Incoterm es utilizado cuando la empresa vendedora entrega la mercancía en el puerto de embarque designado, cuyo riesgo de pérdida o daño a la mercancía se genera cuando la mercancía se encuentra a bordo y, por lo tanto, la empresa compradora corre con los riesgos desde el momento del embarque hasta su llegada al puerto destino, de inicio se considera que todas las operaciones son vía marítima.

En el caso de las importaciones todas las considera CIF (Costo, Seguro y Flete), de acuerdo al Mexico International Chamber of Commerce (2010), significa que la empresa vendedora entrega la mercancía a bordo del buque, el riesgo es el mismo que en el Incoterm anterior, sin embargo cuando este Incoterm es utilizado, significa que la empresa vendedora debe contratar y pagar los costos y el flete necesarios para llevar la mercancía hasta el puerto de destino designado.

El vendedor también contrata la cobertura de seguro contra el riesgo del comprador de pérdida o daño a la mercancía durante el transporte. El comprador debería advertir que, en condiciones CIF, se exige al vendedor que obtenga un seguro con sólo una cobertura mínima. Si el comprador deseara tener más protección del seguro, necesitará acordar cuánta expresamente con el vendedor o bien cerrar sus propios acuerdos extra en cuanto al seguro, este es también un Incoterm para transporte marítimo, sin considerar Incoterms para transporte multimodal o terrestre, generándose desde el inicio inexactitud en la información ya que en el caso de México en 2015 el 62.2\% de las exportaciones y el 50\% de las importaciones se realizó vía terrestre (INEGI, 2015). En el método se considera el volumen, la unidad de medida y el valor unitario, generándose información más exacta, ya que considera el Incoterm utilizado en el pedimento y permite también determinar los volúmenes por fracción arancelaria y unidad de medida, no solo considerando todas las operaciones de importación como FOB, que no toma en cuenta seguros de la mercancía, el flete a destino, gastos de maniobras y despacho aduanal en destino, de esta manera se puede contrastar la 
información no solo a nivel capitulo sino también a nivel partido generándose un nivel de detalle mayor.

El método de validación de la información utilizada para la elaboración de la balanza comercial, para lo siguiente se genera un análisis de cómo se estructura la balanza comercial en los últimos años y los indicadores que de ella se desprenden, de modo que después del análisis, se aplique el método propuesto y con el cual se logre la estandarización de los volúmenes de las mercancías importadas y exportadas en México

De acuerdo al INEGI (2018), en su portal publica como se estructuran los indicadores claves del sector externo y aprobados por la junta de gobierno, se mencionan lo más importantes:

a)

\section{Exportaciones manufactureras}

Se puede definir este indicador como el valor de las mercancías en operaciones realizadas de comercio exterior, las cuales sufrieron un cambio a nivel mecánico, físico, químico a nivel material o de substancia en un proceso de manufactura y que son exportadas legalmente.

Su forma de cálculo es la siguiente:

$$
\left.X M=\sum_{i=1}^{n n} T R_{i}\right)(1)
$$

Significado de las siglas o abreviaturas:

$X M=$ Exportaciones manufactureras

$T R_{i}=$ Transacciones realizadas por concepto de productos $i$ que sufieron una transformación mecánica, física o química de materiales o substancias durante su proceso de producción que se consideran en el Sistema Armonizado. 
$i=$ Productos automotrices; equipos y aparatos eléctricos y electrónicos; maquinaria y equipo especial para industrias diversas; minerometalurgia; alientos, bebidas y tabaco; equipo profesional y científico, entre otros. códigos arancelarios pertenecientes a los capítulos 2; 4; 8;11-24 y 28-97 del sistema armonizado.

Se observa en la fórmula que la unidad de medida es en miles de dólares, por lo tanto, el indicador no ofrece resultados en relación con volumen y está integrado en su cálculo por códigos arancelarios pertenecientes a diferentes capítulos del Sistema Armonizado.

\section{b) Exportaciones petroleras}

Este indicador es el que determinar el valor sin de aquellas operaciones de comercio exterior y en específico de exportación realizadas en alguna de las secciones aduaneras de nuestro país ya sea de productos provenientes de petróleo crudo o del mismo.

Conocer y evaluar el desempeño del intercambio comercial con el resto del mundo a través de la evolución de las exportaciones de mercancías de origen petrolero que coadyuve al análisis y toma de decisiones de los agentes económicos es como define el objetivo de este indicador el INEGI.

Se mide en miles de dólares y su forma de cálculo es la siguiente:

$$
E P=\sum_{i=1}^{n} T R_{\tilde{i}}(2)
$$

Significado de las siglas o abreviaturas:

$$
E P=\text { Exportaciones manufactureras }
$$

$T R_{\tilde{i}}=$ Transacciones realizadas por petróleo crudo y otros productos petroleros $i$ cuyos códigos arancelarios se encuentran considerados en el capítulo 27 de sistema armonizado. 
En su cálculo, considera códigos de un mismo capítulo, pero estos códigos, aunque guardan concordancia por compartir un mismo origen, engloban una cantidad de productos que no siempre son similares.

Este indicador nos permite conocer el total de aquellas operaciones de importación de maquinaria, equipo y bienes que permiten la transformación de materias primas en productos terminados, ya sean de consumo personal o industrial.

Conocer la situación del intercambio comercial de México con el mundo a través de las operaciones de importación de este tipo de bienes, y que ayude a la toma de decisiones acerca de estos temas, es el objetivo de este indicador.

Para su cálculo se utiliza la siguiente fórmula se mide en miles de dólares:

$$
M B K=\sum_{i=1}^{n} O M B K_{i}(3)
$$

Significado de las siglas o abreviaturas:

$M B K=$ Importaciones de bienes de capital.

$O M B K_{i}=$ Operaciones de importación de bienes de capital $i$ con base a los códigos arancelarios del Sistema Armonizado.

Este indicador como los pasados, no considera códigos arancelarios específicos, sino sobre un total de aquellos que son considerados dentro de este rubro.

Importaciones de bienes de consumo

El objetivo de este indicador es las importaciones que realizó nuestro país con el resto del mundo de bienes de consumo y que aporte información para la toma decisiones. 
KOINONIA. Revista Arbitrada Interdisciplinaria de Ciencias de la Educación, Turismo, Ciencias Sociales y Económica, Ciencias del Agro y Mar y Ciencias Exactas y

Se define como el valor total de las importaciones de bienes y productos que satisfacen una necesidad humana y no requieres un proceso adicional y que fueron ingresados al territorio nacional mediante un pedimento de importación.

Se mide en miles de dólares y su fórmula de cálculo es la siguiente:

$$
I B C=\sum_{i=1}^{n} O I B C_{i}(4)
$$

Significado de las siglas o abreviaturas:

$I B C=$ Importaciones de bienes de consumo.

$O I B C_{i}=$ Operaciones de importación de bienes de consumo $i$ con base a los códigos arancelarios del Sistema Armonizado.

El resultado es la mezcla de diversos códigos arancelarios y no se puede determinar un valor por código individual.

e) exportaciones totales

Participación de las exportaciones petroleras en las

Es el porcentaje resultante de dividir las exportaciones petroleras entre las exportaciones totales, permite conocer la participación de las exportaciones petroleras en el total de exportaciones del país en un periodo de tiempo definido, esta información coadyuva al análisis y toma de decisiones en materia económica.

Su fórmula de cálculo se define de la siguiente manera:

$$
P X P_{X T}=\left[\frac{M P}{X T M}\right] 100(5)
$$

Significado de las siglas o abreviaturas:

$P X P_{X T}=$ Participación de las exportaciones petroleras en las exportaciones totales. 
$X P=$ Exportaciones petroleras.

$X T M=$ Exportaciones totales de mercancía.

Por la naturaleza de la información utilizada y el método de cálculo, la información generada es bastante exacta y confiable.

Exportaciones totales.

Este indicador es la suma de todas las operaciones de salida de mercancía de manera legal y por lo tanto mediante un despacho aduanero de exportación, pero contabilizadas para efecto de balanza comercial mediante el Incoterm denominado FOB (Free on Board), tiene como objetivo el conocer y evaluar el desempeño de las exportaciones de nuestro país, mediante el análisis del desarrollo de las exportaciones de mercancías, y que permita a los implicados en la economía la toma de decisiones.

La unidad de medida como los de la mayoría de estos indicadores es miles de dólares y su forma de cálculo se define de la siguiente manera:

$$
\left.X T M=\sum_{i=1}^{n} \operatorname{VOX}_{i}\right)(6)
$$

Significado de las siglas o abreviaturas:

$X T M=$ Exportaciones totales.

VOX $X_{\bar{i}}=$ Valor de cada operación comercial de mercancía $i$ que sale del territorio nacional en un periodo.

Este indicador desde su forma de cálculo presenta errores, mide las operaciones en valor FOB, que es un Incoterm utilizado exclusivamente en operaciones marítimas, por lo cual debería dejar fueras las operaciones que se realizan por otros medios de transporte o distribución, pero no es así, sino que se debe determinar un ajuste para considerar todas las operaciones FOB, pero este no se conoce como se lleva a cabo, generando de esta manera información inexacta (INEGI, 2017). 
KOINONIA. Revista Arbitrada Interdisciplinaria de Ciencias de la Educación, Turismo, Ciencias Sociales y Económica, Ciencias del Agro y Mar y Ciencias Exactas y aplicadas. Año IV. Vol IV. N7. Enero - Junio 2019. Hecho el depósito de Ley: FA2016000010 ISSN: 2542-3088

FUNDACIÓN KOINONIA (F.K). Santa Ana de Coro. Venezuela.

Germán Martínez Prats

Es en estos indicadores claves donde se comienza a existir variaciones en los volúmenes, al solo considerar el valor en dinero, no permite la visualización de los volúmenes como tal, y en algunos casos, menciona las operaciones por fracción arancelarias, pero no se logra visualizar la información a este grado de detalle, sólo se logra visualizar la misma a nivel de capítulo, pero un capítulo puede contener un sinnúmero de fracciones.

\section{RESULTADOS}

A continuación, se presenta la balanza comercial de México en el periodo correspondiente a los años 2012 a 2015 con sus respectivas variaciones. En base a esta información se determinó el modelo de estandarización que puede ayudar a solventar las deficiencias del actual sistema de información.

Tabla 1. Balanza Comercial de Mercancías de México Enero-Junio 2012

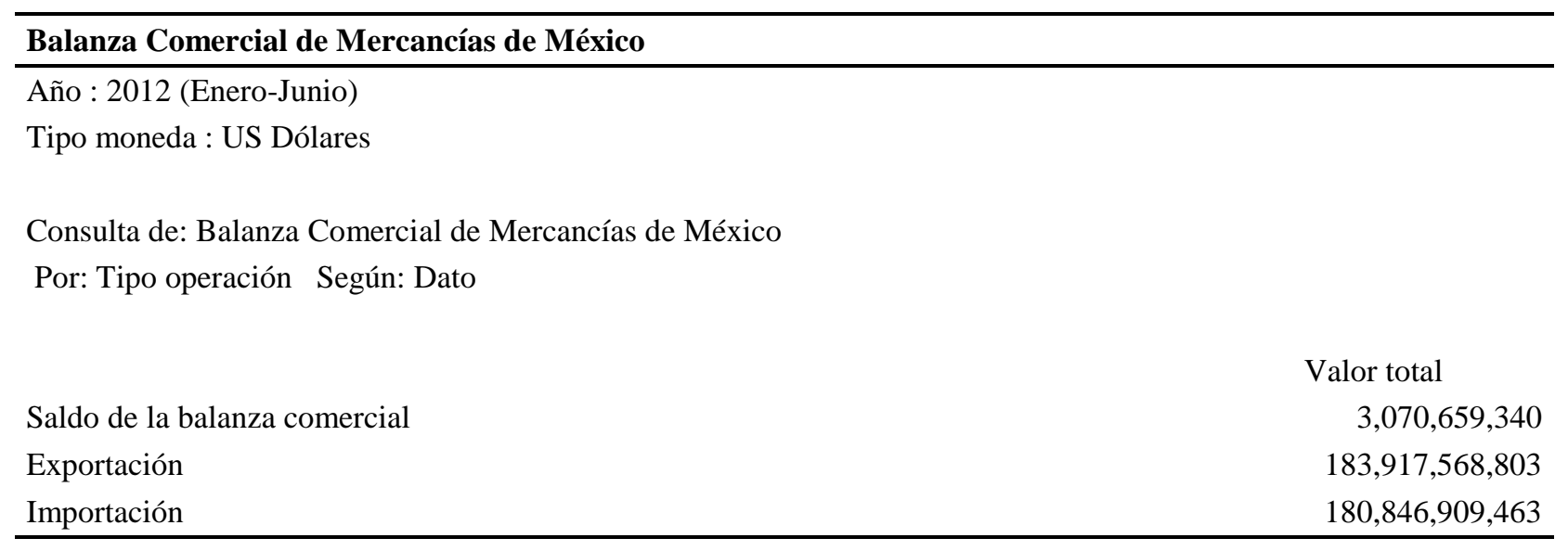

Fuente: INEGI. Estadísticas del Comercio Exterior de México (2017).

Tabla 2. Balanza Comercial de Mercancías de México Julio-Diciembre 2012

\footnotetext{
Balanza Comercial de Mercancías de México

Año: 2012 (Julio-Diciembre)

Tipo moneda: US Dólares

Consulta de: Balanza Comercial de Mercancías de México

Por: Tipo operación Según: Dato
} 
KOINONIA. Revista Arbitrada Interdisciplinaria de Ciencias de la Educación, Turismo, Ciencias Sociales y Económica, Ciencias del Agro y Mar y Ciencias Exactas y aplicadas. Año IV. Vol IV. N7. Enero - Junio 2019. Hecho el depósito de Ley: FA2016000010 ISSN: $2542-3088$

FUNDACIÓN KOINONIA (F.K). Santa Ana de Coro. Venezuela.

Germán Martínez Prats

Saldo de la balanza comercial

Valor total

Exportación

$-3,052,327,905$

Importación

$186,852,321,593$

$189,904,649,498$

Fuente: INEGI. Estadísticas del Comercio Exterior de México (2017)

Tabla 3. Balanza Comercial de Mercancías de México 2013

\section{Balanza Comercial de Mercancías de México}

Año: 2013

Tipo moneda: US Dólares

Consulta de: Balanza Comercial de Mercancías de México

Por: Tipo operación Según: Dato

Saldo de la balanza comercial

Valor total

Exportación

$-1,195,116,291$

$380,015,052,237$

Importación

$381,210,168,528$

Fuente: INEGI. Estadísticas del Comercio Exterior de México (2017).

Tabla 4. Balanza Comercial de Mercancías de México 2014

\section{Balanza Comercial de Mercancías de México}

Año: 2014

Tipo moneda: US Dólares

Consulta de: Balanza Comercial de Mercancías de México

Por: Tipo operación Según: Dato

Saldo de la balanza comercial

Valor total

Exportación

$396,911,688,559$

Importación

$399,977,209,601$

Fuente: INEGI. Estadísticas del Comercio Exterior de México (2017). 
Tabla 5. Balanza Comercial de Mercancías de México 2015

\begin{tabular}{lc}
\hline Balanza Comercial de Mercancías de México & \\
\hline Año: 2015 & \\
Tipo moneda: US Dólares & \\
Consulta de: Balanza Comercial de Mercancías de México & \\
Por: Tipo operación Según: Dato & Valor total \\
& $-14,682,583,649$ \\
Saldo de la balanza comercial & $380,549,784,042$ \\
Exportación & $395,232,367,691$ \\
\hline Importación &
\end{tabular}

Fuente: INEGI. Estadísticas del Comercio Exterior de México (2017).

De acuerdo a Torres (1987) la balanza comercial registra el valor de la totalidad de las importaciones y exportaciones únicamente de mercancías, de un país en un periodo determinado.

En todos los años exceptuando 2012 el saldo de la balanza comercial es negativo, existe un déficit en balanza comercial que indica que se importó más que lo fue exportado, pero el mayor se presenta en 2015 , es allí donde la forma de presentación de la información por parte de las instituciones que la generan es muy escasa, solo se muestran totales, pero no indica cómo se integraron esos totales, es por esta razón que para lograr información a mayor grado de detalle se propone el método de validación. Posteriormente de obtener información de los pedimentos se generó la información de las siguientes tablas, utilizando el método de validación.

Tabla 6. Pedimentos

\begin{tabular}{lllllllll}
\hline FRACCIÓN & $\begin{array}{c}\text { TIPO DE } \\
\text { OPERACIÓN }\end{array}$ & ADUANA & INCOTERM & $\begin{array}{c}\text { VALOR } \\
\text { TOTAL } \\
\text { USD }\end{array}$ & CANTIDAD & $\begin{array}{c}\text { CLAVE } \\
\text { UM }\end{array}$ & $\begin{array}{c}\text { DESCRIPCIÓN } \\
\text { UM }\end{array}$ & $\begin{array}{c}\text { P } \\
\text { UNIT }\end{array}$ \\
\hline 72044999 & IMP & REYNOSA & DAF & $\$ 373.77$ & 16954.207 & 1 & KILOS & $\$ 0.02$ \\
72044999 & IMP & REYNOSA & DAF & $\$ 384.07$ & 17421.415 & 1 & KILOS & $\$ 0.02$ \\
72044999 & IMP & REYNOSA & DAF & $\$ 358.82$ & 16276.075 & 1 & KILOS & $\$ 0.02$ \\
72044999 & IMP & REYNOSA & DAF & $\$ 395.19$ & 17925.818 & 1 & KILOS & $\$ 0.02$ \\
& & & $\$ 1,511.85$ & 68577.515 & & & \\
\hline
\end{tabular}

Fuente: Elaboración propia con datos obtenidos de Servicios Aduanales Castillo SC. 
KOINONIA. Revista Arbitrada Interdisciplinaria de Ciencias de la Educación, Turismo, Ciencias Sociales y Económica, Ciencias del Agro y Mar y Ciencias Exactas y aplicadas. Año IV. Vol IV. N7. Enero - Junio 2019. Hecho el depósito de Ley: FA2016000010 ISSN: $2542-3088$

FUNDACIÓN KOINONIA (F.K). Santa Ana de Coro. Venezuela.

Germán Martínez Prats

Tabla 7. Pedimentos

\begin{tabular}{clllccclc}
\hline \multirow{2}{*}{ FRACCIÓN } & $\begin{array}{c}\text { TIPO DE } \\
\text { OPERACIÓN }\end{array}$ & ADUANA & INCOTERM & $\begin{array}{c}\text { VALOR } \\
\text { TOTAL } \\
\text { USD }\end{array}$ & CANTIDAD & $\begin{array}{c}\text { CLAVE } \\
\text { UM }\end{array}$ & $\begin{array}{c}\text { DESCRIPCIÓN } \\
\text { UM }\end{array}$ & $\begin{array}{c}\text { P } \\
\text { UNIT }\end{array}$ \\
\hline 4069004 & IMP & REYNOSA & DAP & $\$ 67,614.90$ & 18678.15 & 1 & KILOS & $\$ 3.62$ \\
4069004 & IMP & REYNOSA & DAP & $\$ 67,775.63$ & 18722.55 & 1 & KILOS & $\$ 3.62$ \\
4062001 & IMP & REYNOSA & DAP & $\$ 70,489.00$ & 19051.08 & 1 KILOS & $\$ 3.70$ \\
4069004 & IMP & REYNOSA & DAP & $\$ 71,441.55$ & 19051.08 & 1 KILOS & $\$ 3.75$ \\
4069004 & IMP & REYNOSA & DAP & $\$ 71,441.55$ & 19051.08 & 1 & KILOS & $\$ 3.75$ \\
& & & $\$ 348,762.63$ & 94553.94 & & & & \\
\hline
\end{tabular}

Fuente: Elaboración propia con datos obtenidos de Servicios Aduanales Castillo SC..

Tabla 8. Pedimentos

\begin{tabular}{rlllrrrrr}
\hline FRACCIÓN & $\begin{array}{c}\text { TIPO DE } \\
\text { OPERACIÓN }\end{array}$ & ADUANA & INCOTERM & $\begin{array}{c}\text { VALOR } \\
\text { TOTAL } \\
\text { USD }\end{array}$ & CANTIDAD & $\begin{array}{c}\text { CLAVE } \\
\text { UM }\end{array}$ & $\begin{array}{c}\text { DESCRIPCIÓN } \\
\text { UM }\end{array}$ & $\begin{array}{c}\text { P } \\
\text { UNIT }\end{array}$ \\
\hline 2032999 & IMP & REYNOSA & DAF & $\$ 42,621.30$ & 18153.072 & 1 & KILOS & $\$ 2.35$ \\
2032999 & IMP & REYNOSA & DAP & $\$ 42,813.00$ & 18234.72 & 1 KILOS & $\$ 2.35$ \\
2063001 & IMP & REYNOSA & DAP & $\$ 16,650.92$ & 18421.6 & 1 KILOS & $\$ 0.90$ \\
2032999 & IMP & REYNOSA & DAP & $\$ 44,553.60$ & 18289.152 & 1 KILOS & $\$ 2.44$ \\
5040001 & IMP & REYNOSA & DAF & $\$ 25,012.48$ & 9076.53 & 1 KILOS & $\$ 2.76$ \\
2064999 & IMP & REYNOSA & DAF & $\$ 9,745.49$ & 4966.92 & 1 KILOS & $\$ 1.96$ \\
2032999 & IMP & REYNOSA & DAF & $\$ 9,089.56$ & 4123.03 & 1 KILOS & $\$ 2.20$ \\
2032999 & IMP & REYNOSA & DAF & $\$ 39,513.60$ & 18289.15 & 1 KILOS & $\$ 2.16$ \\
2031999 & IMP & REYNOSA & DAF & $\$ 36,326.79$ & 18349.48 & 1 KILOS & $\$ 1.98$ \\
16024999 & IMP & REYNOSA & DAF & $\$ 20,944.00$ & 595 & 1 KILOS & $\$ 35.20$ \\
& & & $\$ 287,270.74$ & 128498.654 & & & \\
\hline
\end{tabular}

Fuente: Elaboración propia con datos obtenidos de Servicios Aduanales Castillo SC.

Se observa en las tablas 4,5 y 6 , el método de validación de los datos que conforman la balanza comercial de México, las cuales permiten la visualización de los volúmenes importados a nuestro país, mediante un documento legal denominado pedimento de importación, de modo que, aplicando ciertas fórmulas, se obtendrá de manera inmediata información que no se presenta INEGI en la balanza comercial, lo que permitirá la estandarización de los volúmenes de las mercancías, categorizándolas por fracción arancelaria, Incoterm, aduana, valor total de la operación, precio unitario, 
unidad de medida y clave de la unidad de medida. Estos datos no son presentados en los documentos de INEGI, pero son visibles en el modelo prestado, como soporte a esto se menciona lo siguiente, la presente investigación se genera por una necesidad que plantea el INEGI en su convocatoria 2015-2 del Fondo Sectorial Consejo Nacional de Ciencia y Tecnología (CONACYT)- INEGI en su demanda 2, donde el objetivo general de esta demanda es desarrollar un modelo o método estadístico para validar y/o ajustar los datos de volumen en una serie de información de al menos cinco años, a partir del cual se genere información de mayor calidad que exprese adecuadamente la realidad de las operaciones comerciales de exportación e importación de mercancías, dicho proyecto no fue asignado a ninguna institución.

Es por esta razón que los resultados presentados en esta investigación no pueden ser contrastado con otros autores por lo menos en el caso de México, no existe información de algún modelo similar, ya que la propia institución encarga de generar esta estadística carece del mismo, es por lo que es ahí donde reside la importancia de este trabajo y justifica la necesidad de un método de este tipo.

Caso contrario a Colombia, en donde se desarrolló una guía para la estandarización de la validación de la información de comercio exterior generada por la Dirección de Metodología y Producción Estadística (DIMPE) de Colombia, el cual se presentó en el XIII Reunión de Expertos Gubernamentales en Estadísticas de Comercio Exterior de Bienes y II Reunión del Grupo Técnico de Calidad de la Información llevado a cabo en Quito, Ecuador. En este documento se relacionaron las prácticas generales del proceso de validación para las variables aplicables en la estandarización de la información de comercio exterior, las cuales se presentan en la tabla 9:

Tabla 9. Variables aplicables para la estandarización de la validación de la información de comercio exterior Código de Aduana (Variable No. 1)

Código de flujo comercial (Variable No. 2)

Régimen (Variable No. 3)

Vía o modo de transporte (Variable No. 6)

Código del puerto o aeropuerto (Variable No. 8)

Código de la naturaleza de la transacción (Variable No. 9) 
Código de condición de entrega (Variable No. 10)

Región de origen o destino (Variable No. 13)

Código de la preferencia (Variable No. 18)

Número de la declaración (Variable No. 4)

Valor estadístico CIF/FOB (Variable No. 21)

Cantidad de la unidad física (Variable No. 24)

Peso neto y Peso bruto (Variable No. 25 y 26)

Fecha de registro (Variable No. 5)

Número de orden de la mercancía de la declaración (Variable No. 11)

Fuente: Elaboración propia con datos de la Dirección de Metodología y Producción Estadística (2007).

En base al análisis de los valores y pesos unitarios por partida arancelaria, se presenta una metodología para controlar la calidad de la información de comercio exterior lo que permite una mayor confiabilidad de la información, así como la automatización en la depuración de la misma. Además, con este método se controla la validación de códigos de las posiciones arancelarias, unidades de medida, peso promedio en kilos admisibles según la unidad de la mercancía, y el valor unitario en dólares para cada posición (Dirección de Metodología y Producción Estadística, 2007).

\section{CONCLUSIONES}

El método de validación propuesto es más exacto en la determinación de la información que debe presentar en la balanza comercial, la información pudiera ser utilizada tanto para la elaboración de la balanza y en la elaboración de los indicadores, permitiendo conocer información a detalle, se pueden clasificar las operaciones a nivel fracción, no solo a nivel capítulo, de esta manera la información generada puede ser de mayor utilidad para los usuarios.

Este método permite una mayor exactitud en la generación de información estadística en materia de comercio exterior, al utilizar una información más detallada los resultados son datos exactos y reales, los cuales a su vez permiten identificar sectores vulnerables dentro de nuestra economía, generándose así áreas de oportunidad que pueden ser atendidas tanto por los empresarios como por las instituciones públicas que 
generan apoyos en materia de comercio exterior, a su vez, permite conocer los sectores en los que tenemos un mayor grado de competitividad a nivel internacional, y de esta manera incrementar en lo posible las condiciones favorables de los mismos.

Como resultado de una información estadística de mayor calidad permite una toma de decisiones rápida y con mayor grado de confiabilidad a todos los actores involucrados en el comercio exterior en México que, de acuerdo a Secretaría de Economía (2010), son más de 30 entre los que encuentran agentes navieros, agentes aduanales, exportadores e importadores.

\section{REFERENCIAS CONSULTADAS}

1. Cardero, E., Mántey, G., \& Mendoza, M. Á. (2006). What is wrong with economic liberalization? The Mexican Case. Investigación Económica, LXV(September), 19-43.

2. Carrillo, J. (2004). Principales estadísticas de la industria maquiladora: encuesta sobre aprendizaje tecnológico y escalamiento industrial. Tijuana, B.C. : Colegio de la $\quad$ Frontera Norte. Retrieved from https://searchworks.stanford.edu/view/5681493.

3. Dirección de Metodología y Producción Estadística. (2007). Guía para la Estandarización de la Validación de la Información de Comercio Exterior. Quito, Ecuador.

4. Instituto Nacional de Estadística y Geografía. (2015). Balanza comercial de Mercancías de México. México. Retrieved from www.inegi.org.mx.

5. Instituto Nacional de Estadística y Geografía. (2017). Balanza Comercial de Mercancías de México. Síntesis metodológica 2017. México. Retrieved from www.inegi.org.mx

6. Instituto Nacional de Estadística y Geografía. (2018). Balanza comercial. Retrieved from http://www.beta.inegi.org.mx/temas/balanza/.

7. Mexico International Chamber of Commerce. (2010). Las nuevas reglas Incoterms 2010. Retrieved from https://www.iccmex.mx/comisiones-detrabajo/incoterms-2010/las-reglas-incoterms. 
KOINONIA. Revista Arbitrada Interdisciplinaria de Ciencias de la Educación, Turismo, Ciencias Sociales y Económica, Ciencias del Agro y Mar y Ciencias Exactas y

8. Moreno-Brid, J. C. (1998). México: crecimiento económico y restricción de la balanza de pagos. Revista Comercio Exterior, 347(5), 1-9. Retrieved from http://revistas.bancomext.gob.mx/rce/magazines/347/5/RCE5.pdf.

9. Secretaría de Economía. (2010). Complejidad del Comercio Exterior. México. Retrieved from http://www.2006-2012.economia.gob.mx/files/Ventanillaunica.pdf.

10.Torres, R. (1987). Teoría del comercio Internacional. México: Siglo XXI editores.

(C2019 por el autor. Este artículo es de acceso abierto y distribuido según los términos y condiciones de la licencia Creative Commons Attribution (http://creativecommons.org/licenses/by/4.0/). 